\title{
Der Fall einer als Stalinismusopfer entschädigten KZ-Aufseherin und weitere Verfahren
}

Es gibt Prozesse, da ist nicht in erster Linie das Urteil von Interesse, sondern es sind die Einzelheiten, Umstände und Hintergründe eines Falles, die sie zutage fördern. Im Gerichtssaal wird nämlich nicht nur Recht gesprochen. Durch die besondere Zwangssituation, der vor allem Zeugen unterliegen, stellt er auch einen einmaligen Ort politischer und historischer Aufklärung dar. So wie in den insgesamt drei Strafprozessen gegen einen Sachbearbeiter der Stiftung für ehemalige politische Häftlinge wegen Vorteilsannahme und die Leiterin der Berliner Gedenkbibliothek für die Opfer des Stalinismus wegen Falschaussage vor Gericht und versuchter Strafvereitelung. Hintergrund war der Fall der als Stalinismusopfer entschädigten ehemaligen SSAufseherin Margot Pietzner, der Mitte der goer Jahre die Phänomene NS-Täterschaft und Kommunismus-Opfertum zusammenbrachte, damit aber einen Nerv der deutschen Gesellschaft traf, zu bundesweitem Aufsehen führte ${ }^{1}$ und schließlich die Szene der SED-Opfer nachhaltig erschütterte. Im Dezember 2000 gingen die juristischen Fragen des Falles zuende. Die politischen bleiben. Begonnen hatte alles zirka zehn Jahre vorher.

Beginnen wir mit dem Fall von Margot P., die 192 I als Margot K. geboren wurde. In den Jahren 1944 und ' 45 hatte sie als Aufseherin der SS im Arbeitslager der Wittenberger Arado-Flugzeugwerke sowie in Außenlagern der KZs Sachsenhausen und Ravensbrück, u.a. in Belzig, Dienst getan. Nach dem Krieg wurde sie von einem sowjetischen Militärtribunal erst zum Tode und dann zu 25 Jahren Haft verurteilt. Bis I $955 \mathrm{saß}$ sie dann fast zehn Jahre in sowjetischen Lagern und DDR-Gefängnissen ein. Danach lebte sie wieder in Wittenberg, wo sie aufgewachsen war. Nach dem Ende der DDR ließ sie sich als politischer Häftling rehabilitieren. Zusätzlich stellte sie einen Antrag auf Haftentschädigung und bekam tatsächlich insgesamt 64350 Mark ausgezahlt. Das war im Frühjahr 1993. Eineinhalb Jahre später wurde der Fall bekannt, führte unter anderem bei ehemaligen KZ-Häftlingen zu heftigen Protesten und machte die Frau quasi zur Person der Zeitgeschichte. Unter dem Druck der Öffentlichkeit mußte der Fall offiziell überprüft werden. Im Sommer I 996 wurde Margot P. der Status als ehemaliger politischer Häftling wieder aberkannt und die Entschädigungssumme von Staats wegen zurückgefordert. ${ }^{2}$

Ein entscheidender Handlungsstrang des Falles führt in die Gedenkbibliothek zu Ehren der Opfer des Stalinismus in Berlin. In der Bibliothek, die im Dezember 1990 eröffnet wurde, sollten Bücher gesammelt werden, die in der DDR verboten waren. Ein vielversprechendes Projekt, das vom Neuen Forum wesentlich unterstützt wurde. Im Vorstand des Bibliotheksvereines engagierten sich bis 1994 bekannte DDR-Bürgerrechtler wie Bärbel Bohley und Jürgen Fuchs. Geleitet wurde die Bibliothek von Anfang an von Ursula P., zu DDR-Zeiten Polnisch-Übersetzerin und keine Oppositionelle oder gar Stalinismusopfer. Da der Ort auch ehemalige DDR-Häftlinge anzog, die etwas andere politische Vorstellungen wie etwa die Bürgerrechtler hatten, kam es schon nach wenigen Jahren zu heftigen politischen Richtungskämpfen, die dann I995 nach dem Bekanntwerden des Falles Margot P.

\footnotetext{
I taz v. I. I2. 1994, Focus 19. 12. 1994

2 dpa-Meldung v. 22.7.1996
} 
zum Auseinanderbrechen der Einrichtung bzw. zum Rückzug der Bürgerrechtler führten. Bärbel Bohley nannte das mit Blick auf die Biografie der Bibliotheksleiterin den "Sieg der Feigen von einst ${ }^{3}{ }^{3}$

Die Details des Falles lagen lange im Dunkeln und wurden erst durch den Prozeß gegen den Behördensachbearbeiter Wolf-Dietrich K. wegen Vorteilsannahme im Januar und Februar 1997 erstmals erhellt. Danach weiß man heute folgendes: Margot P. war im August 1991 in die Gedenkbibliothek gekommen, um ihre Lebensgeschichte vorzustellen, wozu auch die Zeit in der SS gehörte. Nachdem ein Jahr später das neue Strafrechtliche Rehabilitierungsgesetz in Kraft getreten war, halfen ihr Bibliotheksleiterin P. sowie der Schriftsteller Siegmar Faust, der ebenfalls in der Gedenkbibliothek beschäftigt war, bei ihrem Entschädigungsverfahren. Und dabei ging es dann alles andere als korrekt zu. Die zuständige Behörde war die Stiftung für politische Häftlinge, wo auch Sachbearbeiter K. arbeitete, nebenbei ebenfalls Mitglied der Gedenkbibliothek. Obwohl er innerhalb der Stiftungsbehörde gar nicht für Margot P. zuständig war, kümmerte er sich um den Fall. Er benutzte dazu das Dienststellenzeichen des eigentlich zuständigen Sachbearbeiters. Er erfand einen Telefonanruf eines Wittenberger Pfarrers, der mitgeteilt haben soll, Frau P. gehe es sehr schlecht, ob sie nicht bald ihre Entschädigung bekommen könne. Der Antrag sei durch K. beschleunigt worden, erklärte sein Vorgesetzter, der ehemalige Leiter der inzwischen wieder aufgelösten Berliner Stiftungsdienststelle, vor Gericht. K. habe die Antragsformulare rausgegeben, obwohl das entsprechende Gesetz gerade erst in Kraft getreten und überhaupt noch keine Ausführungsbestimmungen erlassen worden waren. Nach den drei Kriterien für die Reihenfolge einer Bearbeitung - Lebensalter, Haftzeit, Schwerbehinderung - hätte der Antrag von Margot P. frühestens ein halbes Jahr später bearbeitet werden dürfen. Durch öffentliche Auftritte des Stiftungsvorsitzenden Roland B. ist allerdings belegt, daß die Stiftung von der Beschleunigung des Verfahrens sehr wohl wußte, sie sogar guthieß. Der Stiftungsvorsitzende nahm seinen Mitarbeiter sogar mehrmals demonstrativ in Schutz. Auch die Bibliotheksleiterin P. war an dem Entschädigungsverfahren beteiligt. Sie hatte den Antrag von Margot P. im März 1993 eigenhändig ausgefüllt; die alte Frau unterschrieb lediglich. Die Bibliotheksleiterin bestritt später mehrfach öffentlich, an dem Antragsverfahren auf $H$ aftentschädigung beteiligt gewesen zu sein. Anfang April 1993 wurde Margot P. schließlich die Entschädigungssumme ausgezahlt. Sie stand auf der sogenannten Sammelliste Nr. I und gehörte zu den ersten, die nach der Einheit Deutschlands ihre Entschädigung bekamen. ${ }^{4}$

Etwa 40000 Mark des Geldes hat Margot P. an alle möglichen Leute verschenkt. Darunter 20000 Mark an Bibliotheksleiterin P. und ihre Familie, wo sie bis zum Zeitpunkt der Auszahlung ein paar Wochen gewohnt hatte, ehe sie in ein Altenheim kam. 7000 Mark gingen an Siegmar F., der nach Bekanntwerden deshalb bei seiner neuen Arbeitsstelle, dem Berliner Landesbeauftragten für die Stasi-Unterlagen, fristlos entlassen wurde. F. wurde später der entsprechende Landesbeauftragte für Sachsen. Und 1000 Mark bekam Sachbearbeiter Wolf-Dietrich K. Auch das wurde mit Verspätung bekannt, führte dann aber zu einem Strafverfahren gegen ihn wegen Vorteilsannahme. Im Januar 1997 kam es so vor dem Kriminalgericht Berlin zum Prozeß Nr. I. Margot P. trat dabei als wichtigste Belastungszeugin auf. Sie erklärte u. a. auch, daß am Vorgang der Geldübergabe Bibliotheksleiterin P. beteiligt war. "Eigentlich darf er nichts nehmen, aber Iooo Mark können wir ihm geben «, habe

3 Der Fall Pietzner und die Gedenkbibliothek, Hörfunksendung SFB I 4. 1. 1995.
4 Prozeßbeobachtung des Autors, Januar/Februar t997.

5 Ebd. 
die damals gesagt. Die Bibliotheksleiterin habe das gesamte Geld verwaltet und ihr dann zwei 500-Mark-Scheine gegeben, die sie K. zusteckte. Ein Detail, das sich mit der Geldübergabe an Siegmar F. deckte. Bibliotheksleiterin P. stritt das ab. Es stand Aussage gegen Aussage. Aber sowohl dem Staatsanwalt als auch dem Richter erschien die Zeugin P. glaubwürdiger. K. wurde wegen Vorteilsannahme zu einer Geldstrafe verurteilt und gegen Bibliotheksleiterin P. nun ihrerseits ein Strafverfahren wegen Falschaussage vor Gericht und versuchter Strafvereitelung eingeleitet. $^{6}$

Im Februar $2000 \mathrm{kam}$ es zum Prozeß gegen Bibliotheksleiterin P. - Prozeß Nr. 2, der im nachhinein wie von der dritten Art erscheint. Es war ein Prozeß mit neuer Rollenverteilung. Diesmal war Wolf-Dietrich K. als Zeuge geladen. Er blieb bei seiner Aussage und bestritt erneut, von Margot P. I000 Mark entgegengenommen zu haben. Was zu diesem Zeitpunkt und noch über den Prozeß hinaus weder die Staatsanwaltschaft noch der Chronist wußten: Margot P., die Belastungszeugin und Hauptfigur, war bereits im September 1998 gestorben. Der Umstand stand zwar nicht in den Ermittlungsakten der Staatsanwaltschaft, aber doch in den Gerichtsakten. Dennoch erklärte der nun befasste Amtsrichter in der Hauptverhandlung, Frau P. habe aus "Gesundheitsgründen «" nicht zum Prozeß nach Berlin anreisen können. So wurde lediglich ihre Zeugenaussage verlesen, die sie vor einem Richter in Wittenberg gemacht hatte. Heute weiß man, vier Tage vor ihrem Tod. Sie war bei ihrer früheren Aussage geblieben, die sie detailgetreu wiederholte. Wieder stand Aussage gegen Aussage. Doch weil sich der Richter, wie er erklärte, kein Bild von der Glaubwürdigkeit der Belastungszeugin machen könne und weil er außerdem den Zeugen $\mathrm{K}$. schonte, der bei einer Verurteilung der Angeklagten P. ein neues Strafverfahren wegen Falschaussage zu erwarten gehabt hätte, urteilte er zugunsten der Bibliotheksleiterin und sprach sie vom Anklagevorwurf frei. P. und $\mathrm{K}$. fielen sich hinterher auf dem Gerichtsflur in die Arme. ${ }^{8}$

Während Prozeß Nr. I noch fünf Tage gedauert hatte, brachte der jetzige Richter Prozeß Nr. 2 in nicht mehr als einer Stunde über die Bühne. Nicht gerade eine Demonstration richterlichen Engagements. Die Staatsanwaltschaft akzeptierte das Urteil allerdings nicht und ging in Berufung. Im November und Dezember 2000 mußte sich Bibliotheksleiterin P. erneut dem Gericht stellen - Prozeß Nr. 3. Die Konstellation, nun vor dem Landgericht Berlin, glich der des vorangegangenen Prozesses. Die Möglichkeiten der Anklagevertretung waren durch den Tod der Belastungszeugin nicht gerade besser geworden, und wieder leitete ein eher lustloser und ungenauer Richter die Verhandlung. Von der Abkürzung SMT für Sowjetisches Militärtribunal beispielsweise - etwas, um das es in der Lebensgeschichre der Margor P. ja ging, das für die Beurteilung ihres Falles eine Rolle spielte und das obendrein in den Akten zu finden ist - wollte der Landrichter noch nichts gehört haben. Das Vexierbild der deutschen Geschichte, die im 20. Jahrhundert vieltausendfach Biografien von Tätern und Opfern hervor- und durcheinandergebracht oder zu Opfertätern und Täteropfern gedoppelt hat und es weiterhin tut, scheint die Justiz zunehmend zu überfordern. Bibliotheksleiterin P. nutzte das aus, um sich wahrheitswidrig z.B. auf den vor zwei Jahren verstorbenen Schriftsteller Jürgen Fuchs zu berufen, der gemeint haben sollte, Margot P. sei von außen in die Gedenkbibliothek eingeschleust worden, um das Ansehen der Einrichtung zu schädigen. Eine Version, die Frau P. bis heute pflegt, um ihre eigene Beteiligung zu verstecken. Daß Fuchs das

6 Ebd.

7 Prozeßbeobachtung des Autors, Februar 2000.

8 Ebd. 
genaue Gegenteil vertreten ${ }^{9}$ und deshalb die Bibliothek verlassen hatte, konnte von diesem Gericht nicht realisiert werden. Am Ende sprach es Bibliotheksleiterin P. erneut frei. ${ }^{10}$

Soweit das Juristische des Falles. Die Strafverfahren haben aber nicht nur die Hintergründe des Falles Margot P. beleuchtet, sondern auch ein Licht auf Politik und Praxis der Entschädigungen für DDR-Häftlinge geworfen, die weit über den Einzelfall hinausgehen. Da ist zunächst das entsprechende Rehabilitierungsgesetz, das 1992 beschlossen wurde und das so verschiedene Häftlingsgruppen wie DDR-Oppositionelle und Bürgerrechtler, Kriegsverwickelte, die möglicherweise NS-belastet sind, oder Mitarbeiter westlicher Geheimdienste in einen Topf wirft. Sachbearbeiter K. zum Beispiel hatte in der Behörde einen Sonderauftrag. Er war für ehemalige Spionagefälle zuständig und bearbeitete die Anträge von Leuten, die für den BND tätig gewesen sind.

Da ist dann die Stiftung für ehemalige politische Häftlinge, die mit den Rehabilitierungsverfahren offiziell betraut war und die den Fall mehr als fragwürdig behandelt hat. Obwohl die SS-Vergangenheit von Margot P. bekannt war, wurde in den einschlägigen Archiven nicht nachgeforscht - weder in der Zentralstelle für die Aufklärung von NS-Verbrechen in Ludwigsburg, noch in der Gauck-Behörde in Berlin, noch im Simon-Wiesenthal-Center in Wien. Erst, nachdem der Antrag positiv beschieden und die Entschädigungssumme von 64000 Mark ausgezahlt, es aber zu öffentlichem Protest gekommen war, holte man das nach. Die Gauck-Behörde stieß bei ihren Recherchen dann auf Dokumente aus den Jahren 1946 bis ' 49 , die in der ehemaligen Kripo-Leitstelle Halle, Außenstelle Wittenberg, sowie in der Kreisdienststelle Wittenberg des MfS lagerten und heute in der Außenstelle der Gauck-Behörde in Halle liegen, und in denen sich nun doch belastende Hinweise fanden. Über die Frau wurde bekannt, daß sie Häftlinge im KZ mißhandelt hatte. Die Stiftungsbehörde hatte sie lediglich aufgrund ihrer eigenen Aussage rehabilitiert, sie sei zur SS dienstverpflichtet worden, sowie aufgrund der Tatsache, daß sie von einem sowjetischen Militärtribunal verurteilt worden war. $\mathrm{Da} ß$ diese Tribunale alles andere als rechtsstaatlich waren, wurde genommen, um die Antragstellerin sozusagen im Umkehrschluß für unschuldig zu erklären. Wenn man die damaligen Vernehmungsprotokolle von 1946 aber genau liest, wie es der Mitarbeiter der Gauck-Behörde Bernd Eisenfeld getan hat, läßt sich durchaus eine differenzierte Bewertung der Schuld der SS-Aufseherinnen erkennen. Entsprechend fielen die Urteile der SMTs - ungeachtet ihrer Rechtsstaatswidrigkeit - unterschiedlich aus. Sie reichten von wenigen Jahren Haft bis zu Todesurteilen, wie ursprünglich auch gegen Margot P. Der Sachbearbeiter, der in der Stiftung für das Verfahren Margot P. eigentlich zuständig war und der ihr die sogenannte $10 / 4$-Bescheinigung nach dem Häftlingshilfegesetz ( $\$ 10 \mathrm{Abs.} 4 \mathrm{HHG}$ ) ausgestellt hatte, mit der sie als politischer Häftling anerkannt wurde, machte vor Gericht eine bemerkenswerte Aussage: Die Recherchen hätten allgemein in der Reihenfolge zu geschehen gehabt, erst nach einer möglichen Stasi-Verstrickung zu forschen und dann eine NS-Verwicklung zu überprüfen. »Mein Gefühl war «, so der ehemalige Sachbearbeiter, der zweieinhalb Jahre in der Stiftungsbehörde arbeitete, wörtlich, "was die Frage des Dritten Reiches anging, sollte man eher ein Auge zudrücken und nicht so ins Detail gehen wie in bezug auf die DDR-Zeit. «" Minde-

9 Jürgen Fuchs, Über die Pietzner/Poprolek/Faust-Geschichte bin ich äußerst empört! Brief vom I2. I. I995, Beilage in: Horch und Guck H. 14/1995.

10 Prozeßbeobachtung des Autors, November/Dezember 2000

i Prozeßbeobachtung (Fn. 4). Vgl. insgesamt Bernd Eisenfeld, Der Entschädigungsfall einer vom sowjetischen Militärtribunal verurteilten SS-Aufscherin, Vortrag auf einer Tagung der Evangelischen Akademie Brandenburg v. 13-5. 1995 
stens einmal ist in der Behörde auch der Rehabilitierungsantrag eines ehemaligen Gestapo-Mannes positiv beschieden worden. Zu diesen Fragwürdigkeiten paßte die Personalpolitik innerhalb der Stiftungsbehörde. Dort wurden Leute eingestellt, die für Verwaltungsaufgaben nicht ausgebildet waren: ein Elektroingenieur, eine Schriftstellerin, K. war Kellner und Lackierer. „Ein Sammelsurium und kunterbunter Haufen«, so der Amtsrichter im ersten Prozeß, der da mit hoheitlichen Aufgaben, dem Auszahlen hoher Geldsummen, beauftragt war. ${ }^{12}$

Schließlich spielte - drittens - auch das Bundesjustizministerium eine etwas seltsame Rolle. Nach der ersten öffentlichen Kritik 1994 stellte es sich vor die Stiftungsbehörde und erklärte in einer offiziellen Stellungnahme, abgesegnet von der damaligen Ministerin Leutheuser-Schnarrenberger, der Fall Margot P. sei korrekt behandelt, die Entschädigung zu Recht gezahlt worden. ${ }^{13}$ Das Ministerium verbreitete dies, obwohl gerade erst die Gauck-Behörde mit Nachforschungen beauftragt worden war und deren Ergebnisse noch nicht vorlagen. Parallel wurde Wolfgang Scheffler vom Zentrum für Antisemitismusforschung an der Technischen Universität Berlin beauftragt zu klären, ob Margot P. freiwillig bei der SS Dienst getan hatte oder ob sie dienstverpflichtet worden war. Schefflers Ergebnis lautete: Die Frage lasse sich nicht klären, beides sei möglich. ${ }^{14}$ Dieser Aussage zum Trotz behauptete das Justizministerium in einem längeren Papier danach, Frau P. sei nicht freiwillig in die SS eingetreten, sondern dienstverpflichtet worden. ${ }^{\text {Is }}$

Anderthalb Jahre später ging der Fall dann doch anders aus. Frau P.'s Rehabilitierung als politischer Häftling wurde rückgängig gemacht; Reste ihrer Entschädigungssumme wurden eingezogen. Die alte Frau hat die Entscheidung nebenbei akzeptiert und selber erklärt, das Geld stehe ihr nicht zu. Von der Summe war allerdings nicht mehr viel übrig. Das Landesversorgungsamt von Sachsen-Anhalt wandte sich auch an Ursula P. und Siegmar F. mit der Bitte, die Geldgeschenke von Frau P. an den Staat zurückzugeben. Eine rechtliche Handhabe, es zurückzufordern, besteht nicht. Die Reaktionen seien gleich null gewesen, so das Amt. Siegmar F. wurde 1996 in Dresden Landesbeauftragter für die Stasi-Unterlagen, 1999 wurde er aber wieder entlassen. Nach wiederholten Auseinandersetzungen u. a. mit Opferverbänden war der Auslöser dann, daß auf seinem Dienstcomputer pornografische Seiten entdeckt wurden. F. will seine 7000 Mark an eine Organisation von Hohenecker Häftlingsfrauen gegeben haben. Bibliotheksleiterin P. hatte nach Bekanntwerden des Falles öffentlich erklärt, sie werde das Geld an Margot P. zurückgeben. Die hatte es obendrein zurückgefordert. Doch das Gegenteil war der Fall. Tage, nachdem die alte Frau ihr die Vollmacht über ihr Konto wieder entzogen hatte, hob P. davon noch mehrere tausend Mark für sich ab. Eine Altenheimbetreuerin berichtete das im Prozeß. Im Gerichtsverfahren gab Ursula P. nun die Version zu Protokoll, sie habe mit dem Geld eine Patenschaft für ein rumänisches Waisenkind übernommen. ${ }^{16}$

Die Gedenkbibliothek für die Opfer des Stalinismus siedelt inzwischen nicht nur im exklusiven Nikolaiviertel in Berlin, sondern bewegt sich auch in einem bestimmten politischen Zusammenhang. Dem der neuen Rechten, zu denen auch alte Rechte gehören: Der ehemalige Bund Freier Bürger, Offensive für Deutschland, die Zeitschriften Deutschland-Magazin und Junge Freiheit, Wehrmachtsoffiziere, Filbingers Studienzentrum in Weikersheim. In diesen Kreisen erlebt der Historikerstreit der

I2 Ebd.

13 Bundesministerium der Justiz, Der Entschädigungsfall Margot Pietzner, Papier, Bonn Februar 1 995 , S. I 2 14.

14 Ebd., S. 9.

is Ebd., S. I4.

16 Eigene Recherchen des Autors sowie Prozeßbeobachtung (Fn. 10). 
8oer Jahre eine neue Blüte - die Argumentation, der Nationalsozialismus sei eine Reaktion zur Verhinderung des Kommunismus gewesen. Einrichtungen wie diese Bibliothek sind dabei Figuren auf dem politischen Schachbrett Deutschland, wo um das Monopol der Aufarbeitung der DDR-Vergangenheit gekämpft wird, um ihre politische Inbesitznahme und wo auch eine Instrumentalisierung von SED-Opfern für die Politik in der Gegenwart stattfindet. Die Schlacht um die »Deutungshoheit über die deutsche Geschichte «7 eben, wie eine Parteivorsitzende zum zehnten Jahrestag der deutschen Einheit einmal sagte.

\section{Wolfgang Hecker \\ Das Verfahren der Wahlprüfung in den Bundesländern*}

\section{Einleitung}

Das hessische Wahlprüfungsgericht hatte am 3. März 2000 die bereits abgeschlossene Prüfung der Landtagswahl in Hessen vom 7. Februar $1999^{\mathrm{I}}$ auf der Grundlage des $₫$ I 8 des hessischen Wahlprüfungsgesetzes (Wahlprüfungs $G$ ) ${ }^{2}$ von Amts wegen wieder aufgenommen. Anlaß waren die bekanntgewordenen »Schwarzgeldkonten « in Höhe von rund 17 Millionen DM, die dem CDU-Landesverband aus im Ausland unterhaltenen Konten zugeflossen waren. Dieses nicht in den Rechenschaftsberichten der CDU deklarierte Vermögen und die Mitfinanzierung des Landtagswahlkampfs von 1999 aus diesen Mitteln waren Anlaß für die Wiederaufnahme des Wahlprüfungsverfahrens durch das Wahlprüfungsgericht. Nach Art. $78 \mathrm{II} \mathrm{HV}^{3}$ machen im Falle der Erheblichkeit für den Ausgang der Wahl eine Wahl ungültig: Unregelmäßigkeiten im Wahlverfahren, strafbare oder gegen die guten Sitten verstoßende Handlungen, die das Wahlergebnis beeinflussen. Das Wahlprüfungsgericht sah in dieser Wahlkampffinanzierung eine sittenwidrige Handlung und Anhaltspunkte dafür, daß das Ergebnis der Landtagswahl dadurch mandatsrelevant beeinflußt wurde.

Nach Auffassung der hessischen Landesregierung war bereits die Einleitung des Wahlprüfungsverfahrens durch das Wahlprüfungsgericht im Wege der Wiederaufnahme nicht mit dem Grundgesetz vereinbar. Auf der Grundlage des Antrags der hessischen Landesregierung im Verfahren der abstrakten Normenkontrolle mußte sich das Bundesverfassungsgericht mit folgenden Fragen befassen:

I. Verstößt Art. 78 II HV, nach dem gegen die guten Sitten verstoßende Handlungen, die das Wahlergebnis beeinflussen, die Wahl im Falle der Erheblichkeit für den Wahlausgang für ungültig machen, gegen das Grundgesetz? Die hessische Landesregierung sah in dieser Bestimmung eine unzulässige Ausweitung der Gründe, die eine Wahl ungültig machen können, und einen Verstoß gegen das rechtsstaatliche Bestimmtheitsgebot.

2. Ist die Zusammensetzung des hessischen Wahlprüfungsgerichts, das gemäß Art. 78

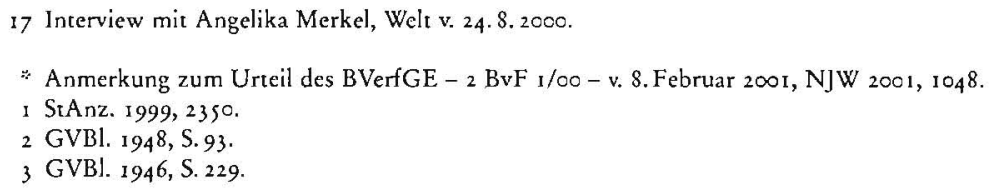

